\title{
Supernovae observations and cosmic topology
}

\author{
M. J. Rebouças ${ }^{1}$, J. S. Alcaniz ${ }^{2}$, B. Mota ${ }^{1}$, and M. Makler ${ }^{1}$ \\ ${ }^{1}$ Centro Brasileiro de Pesquisas Físicas, Rua Dr. Xavier Sigaud 150, CEP 22290-180, Rio de Janeiro, RJ, Brazil \\ e-mail: [reboucas; brunom;martin]@cbpf.br \\ 2 Observatório Nacional, Rua Gal. José Cristino 77, CEP 20921-400, Rio de Janeiro, RJ, Brazil \\ e-mail: alcaniz@on.br
}

Received 28 January 2006 / Accepted 22 February 2006

\section{ABSTRACT}

\begin{abstract}
Context. Two fundamental questions regarding our description of the Universe concern the geometry and topology of its 3-dimensional space. While geometry is a local characteristic that gives the intrinsic curvature, topology is a global feature that characterizes the shape and size of the 3-space. The geometry constrains, but does not dictate, the spatial topology.

Aims. We show that besides determining the spatial geometry, the knowledge of the spatial topology allows us to place tight constraints on the density parameters associated with dark matter $\left(\Omega_{\mathrm{m}}\right)$ and dark energy $\left(\Omega_{\Lambda}\right)$.

Methods. By using the Poincaré dodecahedral space as the observable spatial topology, we reanalyze the current type Ia supernovae (SNe Ia) constraints on the density parametric space $\Omega_{\mathrm{m}}-\Omega_{\Lambda}$.

Results. From this SNe Ia plus cosmic topology analysis, we find best-fit values for the density parameters that are in agreement with a number of independent cosmological observations.
\end{abstract}

Key words. cosmological parameters - cosmic microwave background - cosmology: miscellaneous - methods: miscellaneous cosmology: observations

\section{Introduction}

Cosmologists assume that the Universe can be described as a manifold. Mathematicians characterize manifolds in terms of their geometry and topology. Thus, two fundamental questions regarding our understanding of the Universe concern its geometry and topology. An important difference between these two attributes is that while geometry is a local characteristic that gives the intrinsic curvature of a manifold, topology is a global feature that characterizes its shape and size.

Within the framework of standard cosmology, the Universe is described by a space-time manifold $\mathcal{M}_{4}=\mathbb{R} \times M$ with a locally homogeneous and isotropic Robertson-Walker (RW) metric

$\mathrm{d} s^{2}=-\mathrm{d} t^{2}+a^{2}(t)\left[\mathrm{d} \chi^{2}+f^{2}(\chi)\left(\mathrm{d} \theta^{2}+\sin ^{2} \theta \mathrm{d} \phi^{2}\right)\right]$,

where $f(\chi)=(\chi, \sin \chi$, or $\sinh \chi)$, depending on the sign of the constant spatial curvature $(k=0,1,-1)$. The spatial section $M$ is usually taken to be one of the simply connected spaces, namely, Euclidean $\mathbb{R}^{3}$, spherical $\mathbb{S}^{3}$, or hyperbolic $\mathbb{H}^{3}$. However, this is an assumption that has led to a common misconception that the curvature $k$ of $M$ is all one needs to decide whether the spatial section is finite or not.

In a spatially homogeneous and isotropic Universe, for instance, the geometry, and therefore the corresponding curvature of the spatial sections $M$, is determined by the total matterenergy density $\Omega_{\text {tot }}$. This means that the geometry or the curvature of $M$ is observable, i.e. for $\Omega_{\text {tot }}<1$ the spatial section is negatively curved $(k=-1)$, for $\Omega_{\text {tot }}=1$ it is flat $(k=0)$, while for $\Omega_{\text {tot }}>1 M$ is positively curved $(k=1)$. In consequence, a key point in the search for the (spatial) geometry of the Universe is to use observations to constrain the density $\Omega_{\text {tot }}$. In the context of the standard $\Lambda$ CDM model (which we adopt in this work), this amounts to determining regions in the $\Omega_{\Lambda}-\Omega_{\mathrm{m}}$ parametric plane that consistently account for the observations, and from which one expects to deduce the geometry of the Universe. As a matter of fact, the resulting regions in this parametric plane also give information on the dynamics of the Universe as, for example, whether an accelerated expansion is indicated by the observations, and on the possible behaviors regarding the expansion history of the Universe (eternal expansion, recollapse, bounce, etc.).

However, geometry constrains, but does not dictate, the topology of the 3-manifold $M$. Indeed, for the Euclidean geometry $(k=0)$ besides $\mathbb{R}^{3}$, there are 17 classes of topologically distinct spaces $M$ that can be endowed with this geometry, while for both the spherical $(k=1)$ and hyperbolic $(k=-1)$ geometries there is an infinite number of topologically inequivalent manifolds with non-trivial topology that admit these geometries.

Over the past few years, distinct approaches to probe a non-trivial topology of the Universe $^{1}$, using either discrete cosmic sources or cosmic microwave background radiation (CMBR), have been suggested (see, e.g., the review articles of Lachièze-Rey \& Luminet 1995; Starkman 1998; Levin 2002; Rebouças \& Gomero 2004; Rebouças 2005). An immediate observational consequence of a detectable non-trivial topology of the 3 -space $M$ is that the sky will show multiple (topological) images of either cosmic objects or specific spots of CMBR (Gomero et al. 2001a,b; Weeks et al. 2003; Weeks 2003). The so-called "circles-in-the-sky" method (Cornish et al. 1998), for instance, relies on multiple images of correlated circles in the CMBR maps. In a space with a detectable non-trivial topology, the sphere of last scattering intersects some of its topological images along the circles-in-the-sky, i.e., pairs of matching circles of equal radii, centered at different points on the last scattering sphere (LSS), with the same distribution (up to a phase) of

\footnotetext{
1 In this article, in line with the usage in the literature, by topology of the Universe we mean the topology of the space-like section $M$.
} 
temperature fluctuations, $\delta T$, along the correlated circles. Since the mapping from the last scattering surface to the night-sky sphere preserves circles (Calvão et al. 2005), the correlated circles will be written in the CMBR anisotropy maps regardless of the background geometry and for any non-trivial detectable topology. As a consequence, to observationally probe a nontrivial topology, one should scrutinize the full-sky CMBR maps to extract the correlated circles, whose angular radii, matching phase, and relative position of their centers can be used to determine the topology of the Universe. Thus, a non-trivial cosmic topology is an observable and can be probed for all locally homogeneous and isotropic geometries, without any assumption concerning the cosmological density parameters.

In this regard, the question as to whether one can use this observable to either determine the geometry or set constraints on the density parameters naturally arises. Regarding the geometry it is well-known that the topology of $M$ determines the sign of its curvature (see, e.g., Bernshtein \& Shvartsman 1980). Thus, the topology of the spatial section of the Universe dictates its geometry. At first sight, this seems to indicate that the bounds on the density parameters $\Omega_{\mathrm{m}}$ and $\Omega_{\Lambda}$ arising from the detection of cosmic topology should be very weak, in the sense that they would only determine whether the density parameters of the Universe take values in the regions below, above, or on the flat line $\Omega_{\text {tot }}=\Omega_{\Lambda}+\Omega_{\mathrm{m}}=1$.

In this article, however, we show that, contrary to this indication, the detection of the cosmic topology through the "circlesin-the-sky" method gives rise to very tight constraints on the density parameters. To this end, we use the Poincaré dodecahedral space as the observable topology of the spatial sections of the Universe to reanalyze the current $\mathrm{SNe}$ Ia constraints on the parametric space $\Omega_{\mathrm{m}}-\Omega_{\Lambda}$, as provided by the so-called gold sample of 157 SNe Ia given by Riess et al. (2004). As a result, we show that the knowledge of cosmic topology provides very strong and complementary constraints on the region of the density parametric plane allowed by SNe Ia observations, drastically reducing the inherent degeneracies of current $\mathrm{SNe}$ Ia measurements.

\section{SNe la observations and cosmic topology}

The value of the total density $\Omega_{\text {tot }}=1.02 \pm 0.02$ reported by the WMAP team (Spergel et al. 2003), which favors a positively curved Universe, and the low power measured by WMAP for the CMBR quadrupole $(\ell=2)$ and octopole $(\ell=3)$ moments, have motivated the suggestion by Luminet et al. (2003) of the Poincare dodecahedral space topology as a possible explanation for the anomalous power of these low multipoles. They found that the power spectrum of the Poincare dodecahedral space's fits the WMAP-observed small power of the low multipoles, for $\Omega_{\text {tot }} \simeq 1.013$, which clearly falls within the interval suggested by WMAP. Since then, the dodecahedral space has been examined in various studies (Cornish et al. 2004; Roukema et al. 2004; Aurich et al. 2005a; Gundermann 2005; Aurich et al. 2005b), in which further features of the model have been carefully considered. As a result, it turns out that a Universe with the Poincaré dodecahedral space section accounts for the suppression of power at large scales observed by WMAP, and fits the WMAP temperature two-point correlation function for $1.015 \leq \Omega_{\mathrm{tot}} \leq 1.020$ (Aurich et al. 2005a,b), retaining the standard Friedmann-Lemaître-Robertson-Walker (FLRW) foundation for local physics.

A preliminary search failed to find the antipodal matched circles in the WMAP CMBR sky maps predicted for the Poincaré

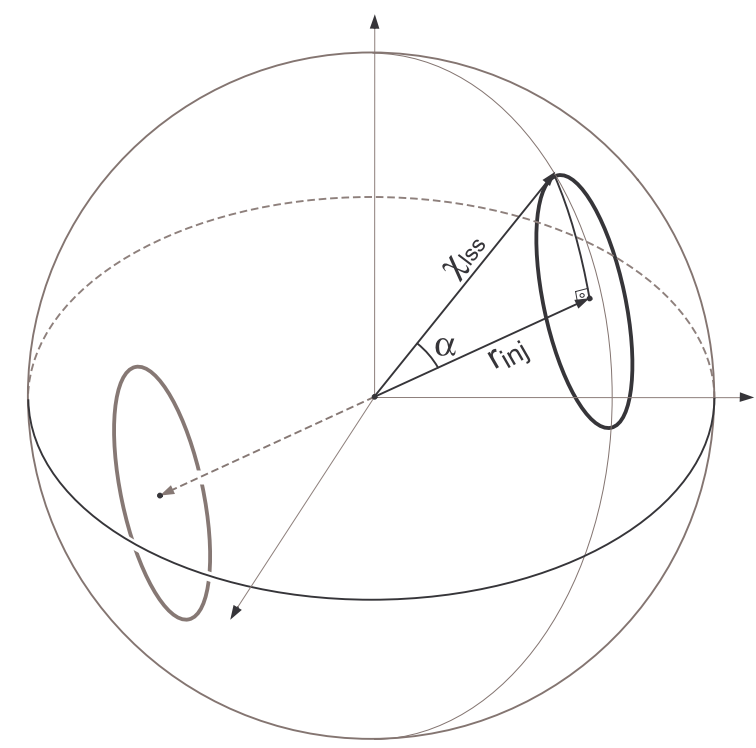

Fig. 1. A schematic illustration of two antipodal matching circles in the sphere of last scattering. The relation between the angular radius $\alpha$ and the angular sides $r_{\mathrm{inj}}$ and $\chi_{\mathrm{lss}}$ is given by the following Napier's rule for spherical triangles: $\sin (\pi / 2-\alpha)=\tan r_{\text {inj }} \tan \left(\pi / 2-\chi_{\text {lss }}\right.$ ) (see, e.g., Coxeter 1965).

dodecahedral space model (Cornish et al. 2004). In a second search, indications for these correlated circles were found, but due to noise and foreground structure of the CMBR maps, no final conclusion has been drawn (Aurich et al. 2005c). We also note that the Doppler and integrated Sachs-Wolfe contributions to the circles-in-the-sky are strong enough to blur the circles, and thus the matched circles can be overlooked in the CMBR sky maps (Aurich et al. 2005a). Additional effects such as the Sunyaev-Zeldovich effect and the finite thickness of the LSS, as well as possible systematics in the removal of the foregrounds, can further damage the topological circle matching.

On these observational grounds, in what follows, we shall assume the Poincaré dodecahedron model.

\subsection{SNe la plus cosmic topology analysis}

To study the consequences of the FLRW model with the Poincaré dodecahedral space section $\mathcal{D}$, we begin by recalling that this model predicts six pairs of antipodal matched circles on the LSS, centered in a symmetrical pattern like the faces of the dodecahedron. Clearly the distance between the centers of each pair of circles is twice the radius $r_{\text {inj }}$ of the sphere inscribable in $\mathcal{D}$. Now, a straightforward use of a Napier's rule to the right-angled spherical triangle with elements $r_{\text {inj }}$, the angular radius $\alpha$ of a matched circle, and the radius $\chi_{\mathrm{lss}}$ of the last scattering sphere (see Fig. 1), furnishes

$\cos \alpha=\frac{\tan r_{\mathrm{inj}}}{\tan \chi_{\mathrm{lss}}}$

where $r_{\mathrm{inj}}=\pi / 10$ for the dodecahedron. Note that $\chi_{\mathrm{lss}}$ depends only on the cosmological scenario, and for the $\Lambda \mathrm{CDM}$ model it reads (in units of the curvature radius)

$\chi_{\mathrm{lss}}=\sqrt{\left|\Omega_{k}\right|} \int_{1}^{1+z_{\mathrm{lss}}} \frac{\mathrm{d} x}{\sqrt{\Omega_{\mathrm{m}} x^{3}+\Omega_{k} x^{2}+\Omega_{\Lambda}}}$,

where $\Omega_{k}=1-\Omega_{\text {tot }}$ and $z_{\text {lss }}=1089$ (Spergel et al. 2003). 
Equations (2) and (3) give the relations between the angular radius $\alpha$ and the cosmological density parameters $\Omega_{\Lambda}$ and $\Omega_{\mathrm{m}}$, and thus can be used to set bounds on these parameters. To quantify this, we proceed in the following way. Firstly, we take the angular radius $\alpha=50^{\circ}$ estimated in Aurich et al. (2005a). Secondly, we note that measurements of the radius $\alpha$ unavoidably involve observational uncertainties, and therefore, in order to set constraints on the density parameters from the detection of cosmic topology, one should take such uncertainties into account. To obtain very conservative results, we take $\delta \alpha \simeq 6^{\circ}$, the scale below which the circles are blurred (Aurich et al. 2005a).

In our statistical analysis, we use SNe Ia data from Riess et al. (2004). The total sample presented in that reference consists of 186 events distributed over the redshift interval $0.01 \lesssim$ $z \lesssim 1.7$ and constitutes the compilation of observations made by two supernova search teams plus, 16 new events observed by the Hubble space telescope (HST). This total data set was initially divided into "high-confidence" (gold) and "likely but not certain" (silver) subsets. Here, we consider only the 157 events that constitute the so-called gold sample. The confidence regions in the parametric space $\Omega_{\mathrm{m}}-\Omega_{\Lambda}$ are determined by defining a probability distribution function $\mathcal{L}=\int \mathrm{e}^{-\chi^{2}(\boldsymbol{p}) / 2} \mathrm{~d} h$, where $\boldsymbol{p}$ stands for the parameters $\Omega_{\mathrm{m}}, \Omega_{\Lambda}$, and $h$, and we have marginalized over all possible values of the Hubble parameter $h$ (for some recent SNe Ia analyses see Choudhury \& Padmanabhan 2003; Nesseris \& Perivolaropoulos 2004; Alcaniz $\&$ Pires 2004). The Poincaré dodecahedral space topology is added to the SNe Ia data as a Gaussian prior on the value of $\chi_{1 \mathrm{ss}}$, which can easily be obtained from Eqs. (2) and (3).

Figure 2 shows the results of our joint $\mathrm{SNe}$ Ia plus cosmic topology analysis. There, we display the confidence regions $(68.3 \%, 95.4 \%$, and $99.7 \%)$ in the parametric plane $\Omega_{\mathrm{m}}-\Omega_{\Lambda}$. Compared to the conventional SNe Ia analysis, i.e. the one with no such cosmic topology assumption (see, e.g., Fig. 8 of Riess et al. 2004), it is clear that the effect of the cosmic topology as a new cosmological observable is to considerably reduce the area corresponding to the confidence intervals in the parametric space $\Omega_{\mathrm{m}}-\Omega_{\Lambda}$, as well as to break degeneracies arising from the current $\mathrm{SNe}$ Ia measurements. The best-fit parameters for this joint analysis are $\Omega_{\mathrm{m}}=0.316$ and $\Omega_{\Lambda}=0.706$ with reduced $\chi_{\min }^{2} / v \simeq$ 1.13 ( $v$ is defined as degrees of freedom). At a $95.4 \%$ confidence level (c.l.) we found $\Omega_{\mathrm{m}}=0.316_{-0.009}^{+0.010}$ and $\Omega_{\Lambda}=0.706 \pm 0.010$, which corresponds to $\Omega_{\text {tot }}=1.022 \pm 0.014$. Note that this value of the total energy density parameter derived from our $\mathrm{SNe}$ Ia plus topology statistics is in full agreement with those reported by the WMAP team, $\Omega_{\text {tot }}=1.02 \pm 0.02$ (Spergel et al. 2003), as well as with the value obtained by fitting the Poincaré dodecahedral power spectrum for low multipoles with the WMAP data, i.e. $1.015 \leq \Omega_{\mathrm{tot}} \leq 1.020$ (Aurich et al. 2005a) and $\Omega_{\mathrm{tot}} \simeq 1.013$ (Luminet et al. 2003).

Concerning the above analysis it is also worth emphasizing three important aspects at this point. First, the range $1.015 \leq$ $\Omega_{\text {tot }} \leq 1.020$ in which the Poincaré dodecahedral space model fits the WMAP data (and also gives rise to six pairs of matching circles) has not been used as a prior of our statistical data analysis. Second, the best-fit values for both $\Omega_{\mathrm{m}}$ and $\Omega_{\Lambda}$ (and, consequently, for $\Omega_{\text {tot }}$ ) depend very weakly on the value used for the angular radius $\alpha$ of the circle. As an example, by assuming $\alpha=11^{\circ} \pm 1^{\circ}$, as suggested in Roukema et al. (2004), it is found that $\Omega_{\mathrm{m}}=0.312_{-0.072}^{+0.078}, \Omega_{\Lambda}=0.698_{-0.078}^{+0.072}$, and $\Omega_{\mathrm{tot}}=$ $1.010 \pm 0.002$ at a $95.4 \%$ (c.l.), which is very close to the value found by considering $\alpha=50^{\circ}$ (Aurich et al. 2005a) with an uncertainty of $6^{\circ}$. Third, the uncertainty on the value of the radius $\alpha$ alters the width corresponding to the confidence regions,

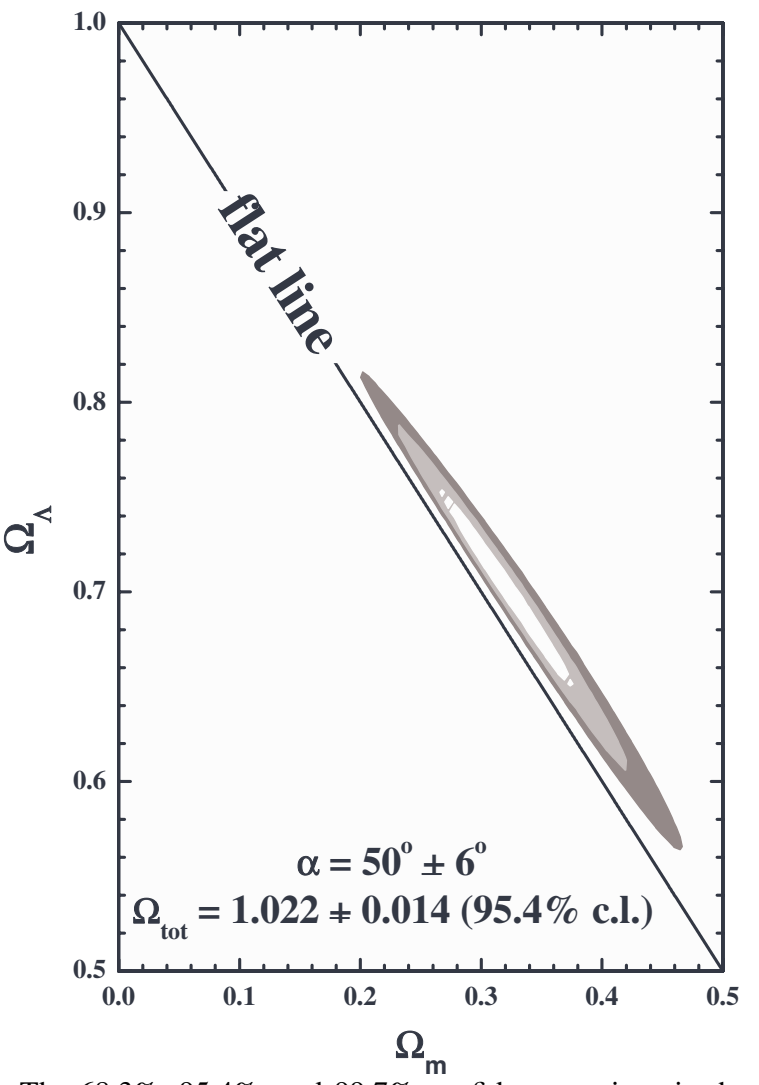

Fig. 2. The $68.3 \%, 95.4 \%$, and $99.7 \%$ confidence regions in the density parametric plane, which arise from the SNe Ia plus dodecahedral space topology analysis. The best-fit values for the dark matter and dark energy density parameters are, respectively, $\Omega_{\mathrm{m}}=0.316_{-0.009}^{+0.011}$ and $\Omega_{\Lambda}=0.706_{-0.009}^{+0.010}$ at a $95.4 \%$ confidence level. The value of the total density parameter, as well as of the angular radius of the circles and the corresponding uncertainties, are also displayed.

without having a significant effect on the best-fit values. Finally, we also notice that, by imposing the topological prior, the estimated value for the matter density parameter is surprisingly close to those suggested by dynamic or clustering estimates (see, e.g., Calberg et al. 1996; Dekel et al. 1997; Feldman et al. 2003; Allen et al. 2004; Pope et al. 2004). On the other hand, as shown in Riess et al. (2004) (see also Choudhury \& Padmanabhan 2003; Nesseris \& Perivolaropoulos 2004; Alcaniz \& Pires 2004), the conventional SNe Ia analysis (without the above cosmic topology constraint) provides $\Omega_{\mathrm{m}} \simeq 0.46$, which is $\sim 1 \sigma$ off from the central value obtained by using independent methods, as for instance, the mean relative peculiar velocity measurements for pairs of galaxies (Feldman et al. 2003).

\section{Final remarks}

Fundamental questions, such as whether the Universe will expand forever or eventually re-collapse and what are its shape and size, are associated with the nature of its constituents as well as with the measurements of both the local curvature and the global topology of the 3-dimensional world. The so-called "circles-inthe-sky" method makes it apparent that a non-trivial detectable topology of the spatial section can be probed for any locally homogeneous and isotropic Universe, with no assumption about the cosmological density parameters. In this article, we have shown that the knowledge of spatial topology of the Universe not only dictates the sign of its local curvature (and therefore its geometry), but also imposes very restrictive constraints on the 
density parameters associated with dark matter $\left(\Omega_{\mathrm{m}}\right)$ and dark energy $\left(\Omega_{\Lambda}\right)$. Indeed, by combining the detection of the cosmic topology through the "circles-in-the-sky" method with the current $\mathrm{SNe}$ Ia observations, we have shown that the effect of the cosmic topology as a cosmological observable is to drastically reduce the degeneracies inherent to current $\mathrm{SNe}$ data, providing limits on the cosmological density parameters, which cannot presently be obtained from combinations of the current cosmological data. This role of cosmic topology has previously been emphasized in the context of cosmic crystallography by Uzan et al. (1999). We underline the fact that the-best fit values are not the most important outcome of our work, since the dodecahedral space model has not been confirmed as the ultimate global topology of the Universe.

We emphasize that even though the precise value of the radius $\alpha$ of the circle and its uncertainty (fundamental quantities in our analysis) can be modified by more accurate analysis and future observations, the general aspects of our analysis remain essentially unchanged, since the best-fit values of the cosmological parameters depend very weakly on $\alpha$, and the value of uncertainty $\delta \alpha$ primarily alters the confidence uncertainty area in the density parametric plane $\Omega_{\mathrm{m}}-\Omega_{\Lambda}$. On the other hand, regarding the possibility of using the observational results to guide the search for the circles in the sky, from a SDSS plus WMAP combination of large-scale structure, SNe Ia, and CMBR data (Tegmark et al. 2004), we can only place an upper bound on the angular radii of the circles for a Poincaré dodecahedral topology, namely $\alpha<70^{\circ}$, which is consistent with value of $\alpha$ we have used in this work.

Given the immense efforts expended in the quest for the local curvature of the Universe, we believe that our results reinforce the cosmological interest in the search for definitive observational evidences of a non-trivial cosmic topology. Further investigations of the other globally homogeneous spherical spaces that also fit current CMBR data are in progress and will be presented in a forthcoming article.

Acknowledgements. The authors are grateful to A.F.F. Teixeira for valuable discussions. We thank CNPq for the grants under which this work was carried out. JSA is also supported by Fundação de Amparo à Pesquisa do Estado do Rio de Janeiro (FAPERJ).

\section{References}

Alcaniz, J. S., \& Pires, N. 2004, Phys. Rev. D, 70, 047303

Allen, S. W., Schmidt, R. W., Ebeling, H., et al. 2004, MNRAS, 353, 457

Aurich, R., Lustig, S., \& Steiner, F. 2005a, Class. Quantum Grav., 22, 2061

Aurich, R., Lustig, S., \& Steiner, F. 2005b, Class. Quantum Grav., 22, 3443

Aurich, R., Lustig, S., \& Steiner, F. 2005c, preprint [arXiv:astro-ph/0510847]

Bernshtein, I. N., \& Shvartsman, V. F. 1980, Sov. Phys. JETP, 52, 814

Calberg, R. G., et al. 1996, ApJ, 462, 32

Calvão, M. O., Gomero, G. I., Mota, B., \& Rebouças, M. J. 2005, Class. Quantum Grav., 22, 1991

Choudhury, T. R., \& Padmanabhan, T. 2003, Mon. Not. Roy. Astron. Soc., 344, 823

Cornish, N. J., Spergel, D. N., \& Starkman, G. D. 1998 Class. Quantum Grav., 15,2657

Cornish, N. J., Spergel, D. N., Starkman, G. D., \& Komatsu, E. 2004, Phys. Rev. Lett., 92, 201302

Coxeter, H. S. M. 1965, Non-Euclidean Geometry (5th ed.; Toronto: University of Toronto Press)

Dekel, A., Burstein, D., \& White, S. D. M. 1997, in Critical Dialogues in Cosmology, ed. N. Turok (Singapore: World Scientific), 175

Feldman, H. A., Juszkiewicz, R., Ferreira, P., et al. 2003, ApJ, 596, L131

Gomero, G. I., Rebouças, M. J., \& Tavakol, R. 2001a, Class. Quantum Grav., 18, L145

Gomero, G. I., Rebouças, M. J., \& Tavakol, R. 2001b, Class. Quantum Grav., 18, 4461

Gundermann, J. 2005, preprint [arXiv: astro-ph/0503014]

Lachièze-Rey, M., \& Luminet, J.-P. 1995, Phys. Rep., 254, 135

Levin, J. 2002, Phys. Rep., 365, 251

Luminet, J.-P., Weeks, J., Riazuelo, A., Lehoucq, R., \& Uzan, J.-P. 2003, Nature, 425,593

Nesseris, S., \& Perivolaropoulos, L. 2004, Phys. Rev. D, 70, 043531

Pope, A. C., Matsubara, T., Szalay, A. S., et al. 2004, ApJ, 607, 655

Rebouças, M. J. 2005, in Cosmology and Gravitation, ed. M. Novello, \& S. E. Perez Bergliaffa (Melville: AIP), AIP Conf. Proc. 782, 188

Rebouças, M. J., \& Gomero, G. I. 2004, Braz. J. Phys., 34, 1358

Riess, A. G., Strolger, L.-G., Tonry, J., et al. 2004, ApJ, 607, 665

Roukema, B. F., Lew, B., Cechowska, M., Marecki, A., \& Bajtlik, S. 2004, A\&A, 423,821

Spergel, D. N., Verde, L., Peiris, H. V., et al. 2003, ApJS, 148, 175

Starkman, G. D. 1998, Class. Quantum Grav., 15, 2529

Tegmark, M., et al. 2004, Phys. Rev. D, 69, 103501

Uzan, J.-P., Lehoucq, R., \& Luminet, J.-P. 1999, A\&A, 351, 766

Weeks, J. R. 2003, Mod. Phys. Lett. A, 18, 2099

Weeks, J. R., Lehoucq, R., \& Uzan, J.-P. 2003, Class. Quantum Grav., 20, 1529 